\title{
Restauro e consolidamento della parte sommitale di castel Penede a Nago (Trento) sul lago di Garda. Un progetto di conoscenza
}

Restoration and consolidation of the top portion of castel Penede in Nago (Trento) on Lake Garda. A project of knowledge

\section{Giorgia Gentilini}

Freelance architect, Trento, Italy, giorgia.gentilini@gmail.com

\begin{abstract}
Castel Penede is located on a rocky spur, the farthest north-west extension of Monte Baldo, protruding to close up the pass from Upper Garda to Adige Valley, protecting the Torbole harbour. The structure of the fortified complex spans over a period of almost five centuries, from the twelfth to the sixteenth century, in an area featuring an at least bi-millennial settlement sequence. The path of knowledge started in 2008 with the historic and stratigraphic analysis. The carrying out of the executive project will turn out to be of a cultural interest, as the removal of the collapsed material will be effected, found in the most ancient rooms which will be then revamped and restored. These works will bring to light some structures (walls, architectonic elements, flooring plans, plasters, vaults and staircases...) which shall be studied, filed and reported as stratigraphic units to update the analysis of the elevations previously carried out. The knowledge methodology adopted to get comprehension of the castle according to scientific methods will be then carried on. The investigations on the architectures have highlighted the relevance of the stratigraphic analysis meant not only as a fundamental aspect of the path of knowledge of a historical building, but also as a basic step to preserve the cultural heritage piece. This will safeguard the readability of the constructed item and of its morphological-stratigraphic connections to the extent of making the traces of the contemporary works on the built item itself both evident and acknowledgeable during the treatment of the walls' surface joints. These operations carried out by skilled workers can be nearly classified as a specialised service which is often not adequately matched in the work practice. It is essential to point out the role of knowledge: knowledge of the item means you know how to restore it.
\end{abstract}

Keywords: Castle, collapsed material, stratigraphic analysis, restoration.

\section{Contesto insediativo}

Il castello di Penede (Fig. 1) occupa l'estremità meridionale del promontorio roccioso di Nago (quota 258 m.s.l.m.) che si protende verso il lago di Garda lungo l'antica strada romana che da Trento giunge a Torbole (Pesavento Mattioli, 2000, p. 19). Il percorso fu strategicamente fondamentale anche in età tardo-antica e medievale (Brogiolo, Azzolini, 2013). Nago è sede di ritro- vamenti archeologici ascrivibili a partire dall'età preistorica (Marzatico, 1988, p. 4; Cavada, 1996, p. 28). L'area, delimitata da una cinta esterna con uno sviluppo di quasi $230 \mathrm{~m}$ e difesa naturalmente da uno strapiombo di $80 \mathrm{~m}$ sui lati est e sud, si estende su circa $3250 \mathrm{~m}^{2}$; al suo interno sono riconoscibili alcuni edifici conservati a diversi gradi di stato ruderale (Gentilini, 2008) che sono stati 


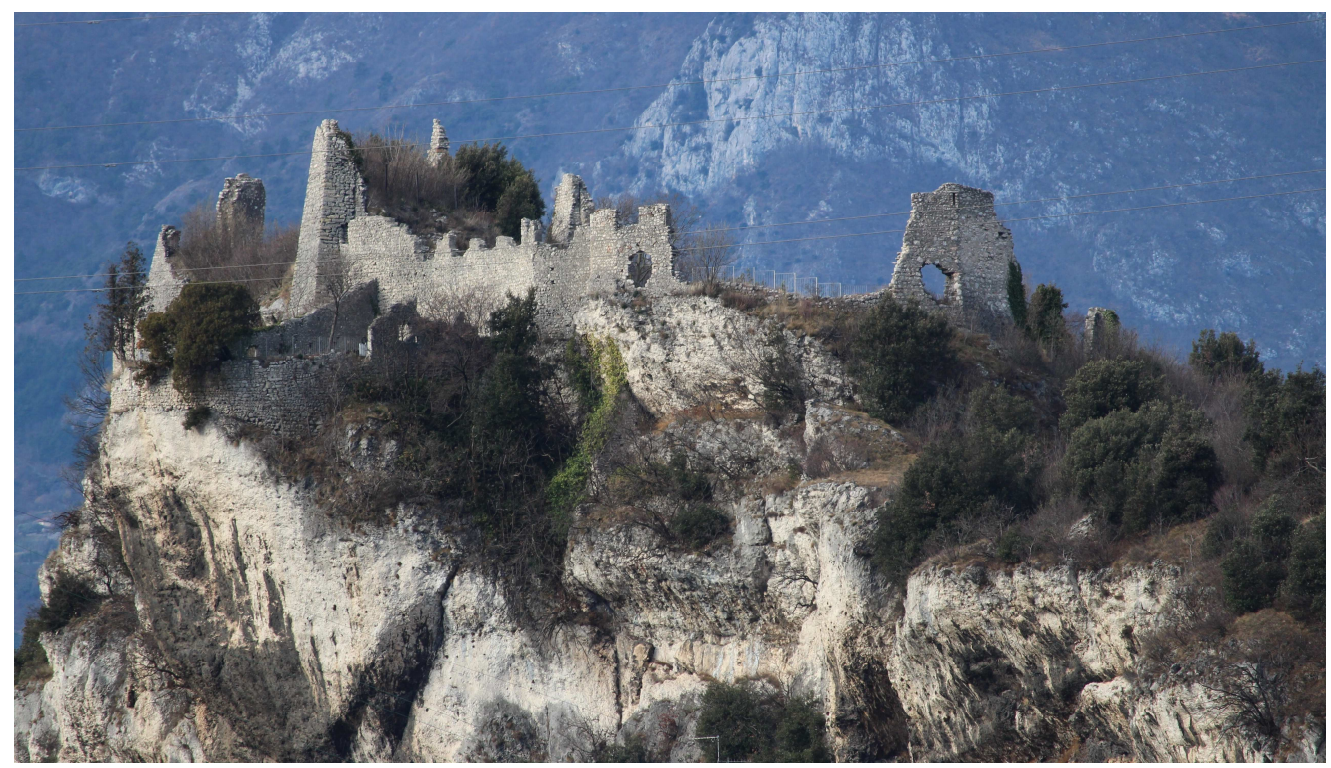

Fig. 1. Vista di castel Penede da nord-est.

identificati e numerati nel corso delle indagini svolte nel 2008, tra i quali un acrocoro con funzione di ridotto signorile e un'ulteriore area insediativa a nord riservata ad attività di servizio.

\section{Inquadramento storico}

Castel Penede (Brogiolo, Gentilini, Landi 2013a) venne eretto da Ulrico II d'Arco (1181-1210) tra il 1203 e il 1207. Il castello viene menzionato la prima volta nel 1210, quando il vescovo Federico Wanga (1207-1218) riportò all'obbedienza gli Arco riuscendo anche ad assicurarsi la sovranità feudale sul maniero. Nei secoli successivi si trovò spesso al centro di lotte e contese. Nel 1266 il castello passò ai Tirolo assieme a tutto il suo patrimonio e da questi ai Castelbarco, nelle cui mani rimase stabilmente fino al 1340 quando Nicolò d'Arco ( $† 1356)$ pose Penede sotto assedio, sostenuto dalle truppe di Luchino Visconti, duca di Milano. I Castelbarco chiesero la protezione del vescovo di Trento. Fu solo nel 1348 che gli Arco riuscirono a strappare la rinuncia al castello e ad ottenere dal vescovado di Trento l'infeudazione con Penede. Il castello passò così agli Arco e vi rimase fino al 1438. Il 16 dicembre di quell'anno il castello, nel contesto delle guerre fra Venezia e Milano, fu occupato dalle truppe del Gattamelata. Il complesso fortificato e la sua giurisdizione pas- sarono pertanto sotto il dominio veneto. Nel contesto della guerra fra l'imperatore Massimiliano I e Venezia maniero e pertinenze furono occupati nel 1509 dalle truppe imperiali e il castello fu restituito, questa volta come feudo imperiale, agli Arco. Questa situazione nel corso del XVI secolo provocò diversi attriti con Ferdinando I d'Austria, quale conte di Tirolo; tale condizione esplose al tempo delle lotte fratricide degli Arco a metà del XVI secolo. Il governo enipontano decise quindi di sfruttare l'occasione, anche con il pretesto di ripristinare la pace e l'ordine in quella regione, e nel 1579 occupò Arco e Penede, insediandovi propri capitani. Il governo tirolese di Castel Penede durò fino al 1614, quando il castello venne restituito -questa volta come feudo tirolese- ai conti d'Arco, che riuscirono a tenerlo fino al 1672, quando fu loro tolto per la cattiva gestione. Nel 1681 il castello ritornò però agli Arco e rimase nelle loro mani fino al 1703, quando fu assediato e diroccato dalle truppe francesi nel contesto della guerra di successione spagnola. Da allora il castello è in rovina. Nel 1986 è diventato di proprietà del Comune di Nago-Torbole.

\section{La sequenza costruttiva}

Il progetto di conoscenza è iniziato nel 2008 attraverso l'analisi storica e stratigrafica, con la 
quale sono stati riconosciuti quattro periodi costruttivi principali, in un arco temporale di circa sette secoli (Fig. 2).

\subsection{Periodo I (fine XII-XIII secolo)}

In questo periodo vengono raggruppate le tecniche murarie ritenute romaniche, caratterizzate da utilizzo di bozze in calcare bianco disposte in opera con una certa cura e in corsi regolari. Sono documentate sia nella cinta esterna, sia in due edifici sommitali (CF I, CF II). La datazione tra fine XII e XIII secolo è suggerita dalla tecnica muraria e dalla tipologia di alcune aperture superstiti caratterizzate da cornici monolitiche in pietra squadrata sempre calcarea. L'analisi stratigrafica induce a ricondurre le murature romaniche degli edifici sommitali a due distinte architetture. Del corpo di fabbrica I si riconoscono alcune strutture. In sezione, stretta tra due murature che la inglobano, l' angolata sud-ovest, si conserva in alzato per 7-8 $\mathrm{m}$ ed è caratterizzata da conci squadrati in calcare bianco con corsi regolari. Nel cunicolo aperto in rottura tra la cisterna e l'osservatorio militare S, si rilevano, in calcare, lo stipite e l'architrave di una porta in pietre monolitiche. Non è invece documentato il rapporto tra CF I e l'edificio II nella cui direzione si sviluppa. Di quest'ultimo, la vera e propria residenza signorile del castello, sono osservabili alcune murature. L' angolo sud-ovest, conservato per alcuni metri in pianta e per tre piani in alzato (solo lato ovest), è in calcare bianco bugnato. L'analisi al radiocarbonio con spettrometria di massa ad alta risoluzione (AMS) di un campione di calcinarolo presente nella malta in questo punto (Miani, 2011), ha prodotto datazione: 1221 AD - 1291 AD $(95,8 \%)$. I paramenti delle pareti sono in conci squadrati di calcare bianco nelle facce esterne, in bozze spaccate in quelle interne, in entrambi messi in opera con regolarità. Si documentano inoltre un tratto di poco più di un metro del lato est e resti di un probabile divisorio. L'ambiente meridionale (UF 03) dell'edificio era voltato a botte (se ne conserva l'attacco nel lato sud). Nella parete ovest, si trovano tre finestre, una per ciascun piano; caratterizzate nella fase più antica da cornici squadrate in pietra calcarea grigia Corno di Bò, rimasero in uso anche dopo la foderatura di rinforzo del periodo III.

\subsection{Periodo II (fine XIII-XIV secolo?)}

Fase I

Il periodo II, documentato solo nella cinta esterna, corrisponde ad una ricostruzione della cortina e alla realizzazione del "rondello est"; è marcato sia da paramenti in opera incerta in pietre calcaree spaccate di piccola dimensione e a spigoli vivi, sia da grandi merlature a coda di rondine, delimitate da lastre di pietra verticali. Queste ultime sono rivestite da un intonaco a falsa cortina incisa con lisciature semplici a cazzuola, che imita l'apparato dei paramenti romanici, particolare che ne suggerisce una datazione quantomeno all'avanzato XIII secolo (se non più tardi), che si ritiene possa valere anche per quelle della grande cinta esterna. Nel tratto S inoltre, a quota elevata e verso est, una muratura in opera incerta è in fase con i merli a coda di rondine. In quello est, la muratura osservata, è in fase con i merli e con la porta che mette in comunicazione l'interno con il "rondello". Di questo si conservano in alzato un tratto verso nord con due merli della medesima tipologia e una feritoia e quello sud, dove si trovano altre tre feritoie. Nel settore nord della cinta esterna, si conserva un solo brano murario che presenta una tecnica in opera incerta e una marcata rientranza per il camminamento di ronda difeso a muro continuo nel quale si apre una feritoia.

\section{Fase II}

La muratura a nord della porta, con arco a sesto ribassato che dà accesso al "rondello est", presenta una tessitura diversa rispetto alla porzione verso sud sopra descritta; diversi sono anche i merli soprastanti e soprattutto, la struttura della cinta sormonta la muratura del "rondello est". Se ne deve dedurre che in questo tratto è stato rifatto viste anche altre peculiarità osservabili nel prospetto interno, quali una scala di accesso al piano superiore alla quota del camminamento di ronda; una serie di mensole in pietra calcarea di sostegno ad una travatura dormiente e poi sostituite da una serie di fori per alloggio di travature orizzontali; un'ampia finestra ad arco ribassato con stipiti in pietra calcarea squadrata che pre- 
senta due sedili laterali; una latrina ricavata nel sottoscala e con scarico all'esterno della cinta tramite un buco verticale. Tutti questi elementi fanno ipotizzare che alla cortina. in questa fase fosse stato addossato un corpo di fabbrica $(\mathrm{CF}$ IX).

\subsection{Periodo III (XV secolo)}

Sia la cinta esterna sia gli edifici sommitali dell'acrocoro vengono rinforzati con imponenti foderature scarpate, realizzate con la funzione di protezione dal tiro delle artiglierie. Nel lato nord del "rondello est", una muratura di sopraelevazione copre i merli ed è forse in fase con una finestra rettangolare. A questo momento è plausibilmente riferibile anche la ristrutturazione interna degli edifici I e III che comporta: una rifoderatura della parete sud dell'edificio I con il mantenimento di due aperture preesistenti; la costruzione di una cisterna sempre all'interno dell'edificio I (UF 01); e, infine, la costruzione di un vano voltato (UF 02 con porta di accesso e intonaco a base di calce idraulica con cocciopesto) nell'edificio III.

\subsection{Periodo IV (post XV secolo)}

Del castello documentato nella pianta nel 1615 (Fig. 3) non si conservano altre evidenze fuori terra se non quanto in precedenza descritto. $\mathrm{Si}$ stima che il castello, danneggiato nelle operazioni militari del 1703, sia stato poco dopo abbandonato.

Le operazioni successive si possono identificare in fasi di crollo con formazione di consistenti depositi archeologici nella parte sommitale e fasi di spoliazione della cortina più esterna, nei tratti ovest e nord. Si ritiene che, in un momento ancora successivo, sia avvenuto un riutilizzo di alcuni edifici, in particolare il V e VI, le cui murature sono ancora conservate per molti metri in alzato; una riduzione a coltura dello spazio interno al rondello est, caratterizzato da un riporto di terra perfettamente orizzontale; la rimozione di parte del crollo nel settore settentrionale. Il castello è stato infine oggetto di due distinte operazioni di restauro: la prima caratterizzata da rifacimenti non facilmente riconoscibili rispetto alle fasi storiche di vita del castello; la seconda operata dalla Soprintendenza per i Beni Culturali nel 1994-1995, con integrazione di lacune trami-

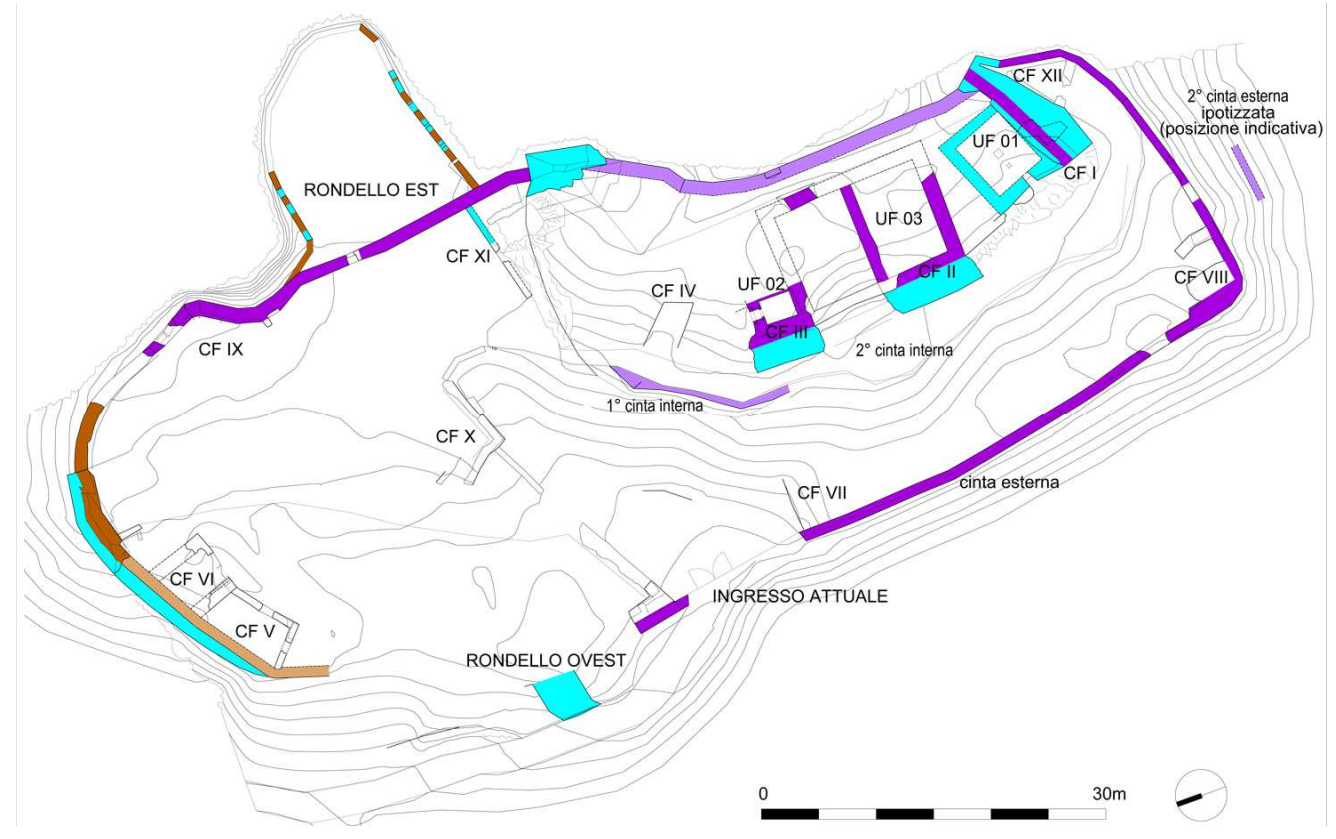

Fig. 2. Planimetria con i periodi costruttivi. 


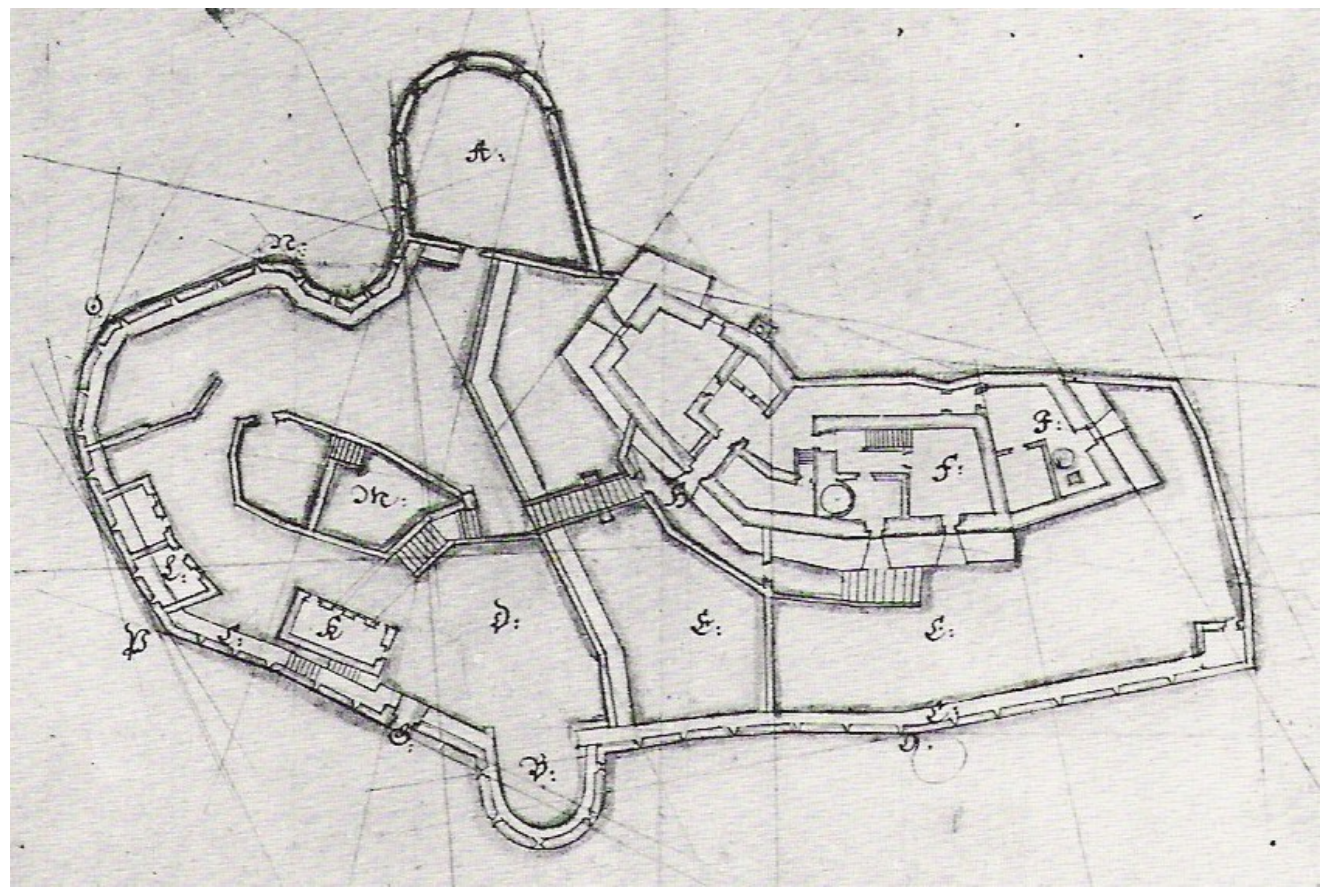

Fig. 3. Particolare della planimetria di castel Penede del 1615 (Innsbruck, Tiroler Landesarchiv, Handschriften, Bd. 3, 19. Rasmo N., Der Innsbrucker Kodex III und die Tiroler Landesverteidigung gegen Venedig im Jahre 1615, Trient 1979).

te murature sottosquadro e un'ampia rifugatura delle malte con obliterazione, in alcuni casi, del dato stratigrafico.

\section{Conoscere per restaurare}

Da questo presupposto prende vita il progetto di recupero di Castel Penede, intrapreso dal Comune di Nago-Torbole, proprietario del maniero, con il supporto e l'assistenza della Soprintendenza per i Beni Culturali della Provincia autonoma di Trento. L'operazione di conoscenza, iniziata nel 2008, che sta continuando con il cantiere attualmente in corso, costituisce una nuova sfida per l'edificio fortificato. L'obiettivo è mettere in sicurezza la parte sommitale del castello, le cui murature interne e perimetrali sono degradate e ricoperte da materiale di crollo. Lo scavo, sorvegliato dagli archeologi, sta mettendo in luce murature, elementi architettonici, piani pavimentali, intonaci, volte e scale che componevano gli ambienti riconoscibili grossomodo nella planimetria del 1615.

Siamo nel pieno di un lavoro delicato da svolgere nel rispetto della stratigrafia secondo le più avanzate metodologie scientifiche per garantire la conservazione del bene culturale e la sua futura fruibilità. Allo stato attuale le operazioni di rimozione del materiale di crollo presente (a mano e con piccoli mezzi) si sono concentrate nei Corpi di Fabbrica I, II (Figg. 4 e 5), III e in parte nel IV, sotto costante assistenza archeologica.

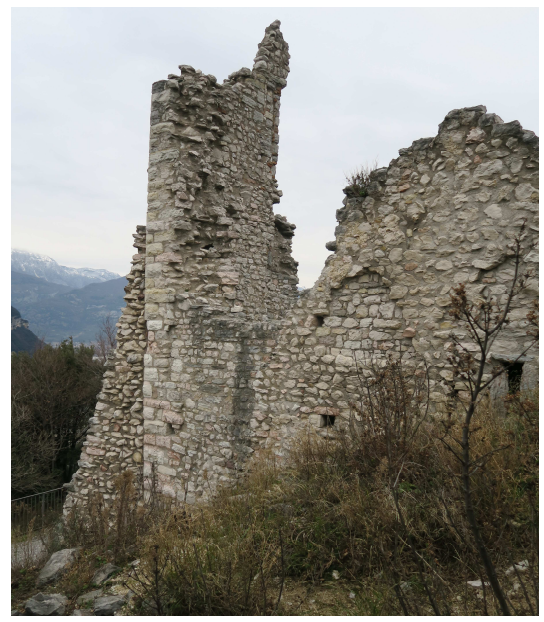

Fig. 4. CF II UF3, prospetto sud esterno (02/2018). 


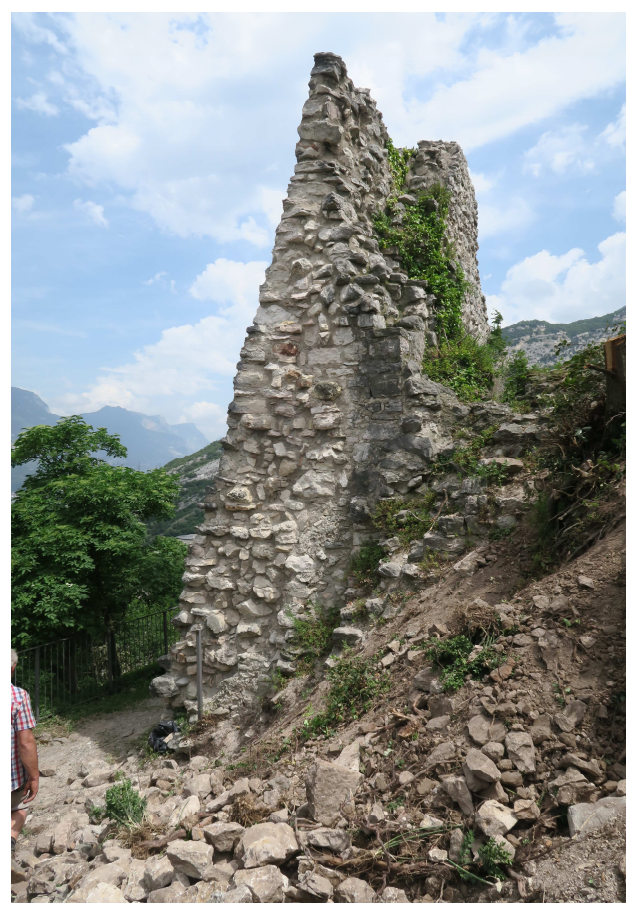

Fig. 5. CF II, prospetto sud interno inizio lavori (05.2019).

Questa operazione è iniziata dopo aver eseguito il disboscamento dell'area e verificato le creste murarie delle vele conservate e fissato elementi smossi per la sicurezza e l'incolumità degli operatori. Il primo ad essere stato messo in luce è stato, seppure parzialmente, il CF IV con l'identificazione del vano d'ingresso, in salita, agli edifici residenziali presenti sull'acrocoro (UF04), caratterizzato da una pavimentazione con gradini in pietra calcarea, e dalla parete sud intonacata che ingloba un'angolata precedente (Fig. 6) e conserva anche qualche traccia di affresco. Si entra quindi nel UF05, un ambiente voltato, di distribuzione, con una scala (Fig. 7) che portava al piano superiore; poi nel vano UF06 a nord-ovest attiguo all'UF02, due ampi portali in pietra Corno di Bò (di cui si conservano in situ i piedritti) che davano accesso a nordest al grande ambiente del CF IV e al CF II (Fig. 8) con le sue due unità funzionali; sempre da UF05 si sviluppa ad E il passaggio, perimetrato dalla cinta esterna a strapiombo, che conduceva all'edificio CF I.

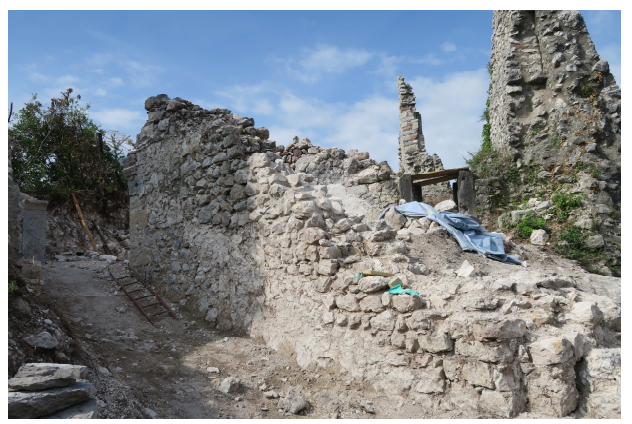

Fig. 6. CF IV UF 4, prospetto sud ingresso (08/2019).

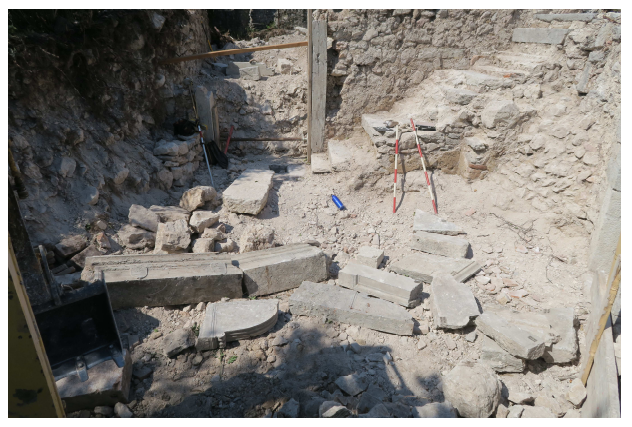

Fig. 7. CF IV UF5, vista verso sud durante i lavori.

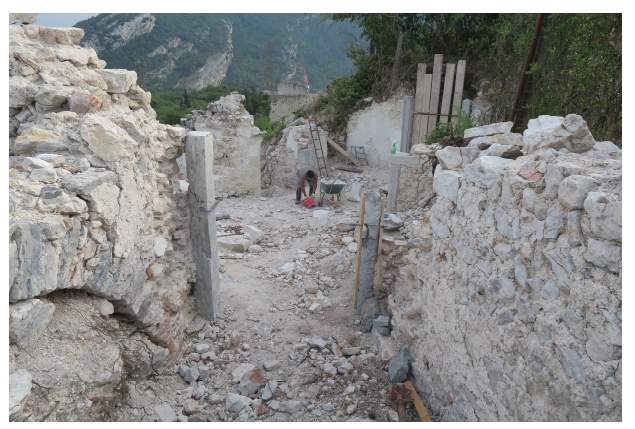

Fig. 8. Vista dell' UF 5 da CF II durante i lavori.

Di entrambi i portali sono stati trovati e schedati gli architravi, di cui uno conserva scolpiti gli stemmi delle famiglie Arco, Malaspina e Vels. Questo ambiente mantiene una pavimentazione in lastre di pietra calcarea che verrà messa in luce e restaurata appena completato lo scavo archeologico degli ultimi $30 \mathrm{~cm}$ sopra pavimento. Attraverso il CF IV si entra nel CF II: qui è stata rinvenuta un'altra scala a servizio dell'edificio. La prima nel CF IV appoggia su una muratura e i pochi gradini conservati sono in pietra calcarea grigia in Corno di Bò; la seconda è impostata su 
un arco rampante (Fig. 9). Da questo ambiente si saliva ai piani superiori, si entrava nell'UF 7 e in fondo all'UF 3.

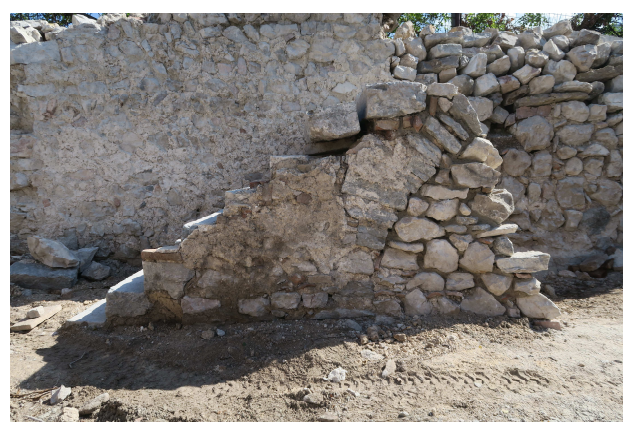

Fig. 9. CF II UF 7, scala in pietra su arco rampante.

Di quest'ultima, prima dello scavo, era visibile la struttura voltata crollata nell'angolo sud-ovest; la rimozione del materiale di crollo (Fig. 10) ha messo in evidenza che la struttura a botte è stata inserita in rottura nella muratura romanica del CF II in quanto chiude la feritoia presente (Figg. 4 e 10); qui inoltre non è stato rinvenuto un pavimento finito ma direttamente la roccia; dopo lo scavo archeologico vedremo se è stata lavorata per adattarla ad un piano calpestabile. Anche qui per le informazioni definitive si deve aspettare l'indagine archeologica finale dell'ultimo strato di materiale.

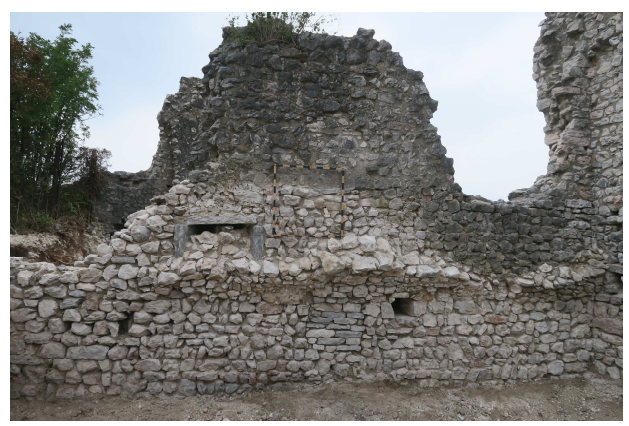

Fig. 10. CF II UF 3, prospetto interno sud dopo la rimozione.

La seconda unità funzionale del CF II presenta un'interessante stratificazione sul prospetto nord interno (Fig. 11): sulla facies interna della muratura perimetrale è stata rinvenuta la traccia di un camino/stufa ad olle con la sua canna fumaria poi rotta e in parte tamponato per la realizzazione di una struttura a volta a crociera con imposta molto bassa ri- spetto alla soglia di ingresso. Il proseguo dello scavo potrà chiarire meglio la questione delle quote, l'andamento e le caratteristiche del piano pavimentale dell' ambiente.

Completata la prima parte di rimozione del materiale di crollo con assistenza archeologica, ora si sta procedendo nell'aggiornamento dell' analisi stratigrafica degli alzati per poter dare indicazioni precise all'impresa che eseguirà il consolidamento ed il restauro delle murature e degli intonaci rinvenuti al fine di garantire il mantenimento dell'Identità stratigrafica .

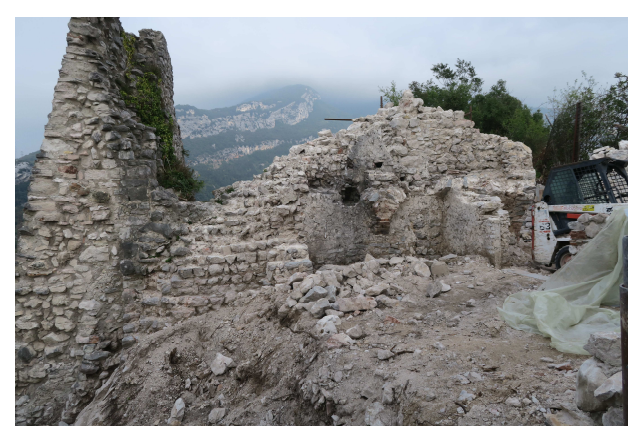

Fig. 11. CF II UF 7, scala in pietra su arco rampante.

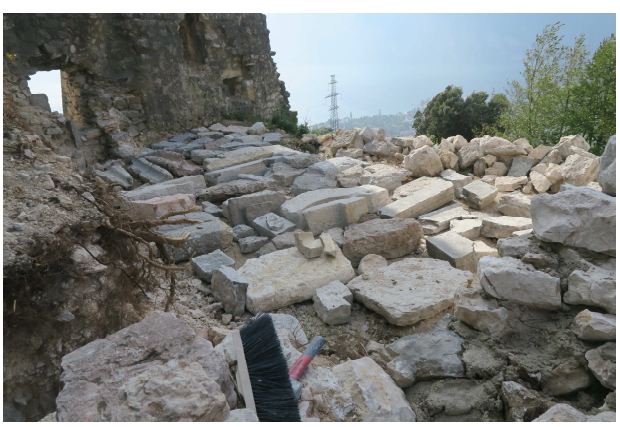

Fig. 12. CF I, alcuni degli elementi architettonici rinvenuti.

Per quanto riguarda le integrazioni murarie, essendo la muratura antica in pietre calcaree sbozzate con giunti rifluenti, si è proceduto selezionando gli elementi lapidei disponendoli con una tessitura più di fascia che di testa, su corsi suborizzontali e con un leggero sottosquadro per una leggibilità calibrata del nuovo intervento, che aiuti nella lettura delle fasi costruttive soprattutto dove la malta antica non è conservata e i nessi stratigrafici potrebbero venire sfalsati con l'uso di un'unica malta di restauro per la risarci- 
tura e la finitura dei giunti. Quest'ultima è stata realizzata con un impasto composto da calce idraulica naturale ed inerti ponderati sull'esistente, con l'aggiunta di sabbia di colore nero (diametro 1-2 $\mathrm{mm}$ ).

\section{Conclusioni}

Le nuove strutture murarie, gli intonaci che coprono i giunti lisciati a scivolo, i pavimenti in pietra calcarea e in malta di calce aerea, i reperti rinvenuti (ceramica, una fibbia, uno sperone in ferro, frammenti di affresco,...), i numerosissimi elementi architettonici (architravi, capitelli, mensole, doccioni, lastre,...) sono la testimonianza della cultura materiale di castel Penede: all'interno di un involucro romanico ed una rifodera tardo quattrocentesca, si conservano ambienti che, in base anche allo studio araldico sugli stemmi rinvenuti, portano ad ipotizzare una ristrutturazione attorno agli anni 30 del XVI se- colo, dopo la restituzione di castel Penede agli Arco, con nuovi dati per il Periodo IV. Ma siamo ancora in cantiere e non sono ancora concluse tutte le indagini di questo primo lotto.

\section{Note}

Il progetto esecutivo è stato redatto dalla sottoscritta su incarico del Comune di Nago-Torbole nell'agosto 2018 con la consulenza della restauratrice di beni culturali Monica Endrizzi. Il cantiere, iniziato il 20 maggio 2019, si concluderà a fine novembre 2019; l'impresa esecutrice Tecnobase srl (Trento) sta eseguendo i lavori con la DL della sottoscritta, la sorveglianza archeologica della ditta ARC-TEAM snc (Cles, TN). Per la Soprintendenza per i Beni Culturali della Provincia autonoma di Trento sono presenti i funzionari dott.ssa Nicoletta Pisu (Uff. Beni archeologici) e arch. Cinzia D’Agostino (Uff. Beni architettonici).

\section{Bibliography}

Brogiolo, G.P.; Azzolini, A. (2013). "Fortificazioni e chiuse nella Val d'Adige", in Possenti, E.; Gentilini, G.; Landi, W.; Cunaccia, M. eds., Castra, castelli e domus murate. Corpus dei siti fortificati trentini tra tardo antico e basso medioevo: Saggi, Apsat 6, SAP Mantova, pp. 41-60.

Brogiolo, G.P.; Gentilini, G.; Landi, W. (2013a). "Castel Penede a Nago nel Sommolago", in Possenti, E.; Gentilini, G.; Landi, W.; Cunaccia, M. eds., Castra, castelli e domus murate. Corpus dei siti fortificati trentini tra tardo antico e basso medioevo: Saggi, Apsat 6, SAP Mantova, pp. 217-248.

Brogiolo, G.P.; Gentilini, G.; Landi, W. (2013b). "Nago Castel Penede", in Possenti, E.; Gentilini, G.; Landi, W.; Cunaccia, M. eds., Castra, castelli e domus murate. Corpus dei siti fortificati trentini tra tardo antico e basso medioevo: Saggi, Apsat 6, SAP Mantova, pp. 417-425.

Cavada, E. (1996). "In Summolaco: continuità o discontinuità dell'insediamento", in Brogiolo, G.P. ed., La fine delle ville romane: trasformazioni nelle campagne tra tarda antichità e alto medioevo, Atti del Convegno (Gardone Riviera, 14 ottobre 1995), Mantova, pp. 21-34.

Doglioni, F. (1997). Stratigrafia e restauro: tra conoscenza e conservazione dell'architettura, Lint Editoriale Associati, Trieste.

Doglioni, F. (2008). Nel restauro. Progetti per le architetture del passato, Marsilio, Venezia.

Doglioni, F. (2010). "Leggibilità della costruzione, percorsi di ricerca stratigrafica e restauro", Archeologia dell'Architettura, XV, pp. 65-79.

Gentilini, G. (2008). Ricerca storico-iconografica e analisi stratigrafica delle strutture del complesso fortificato di castel Penede a Nago, (inedito, Archivio Comune di Nago-Torbole), Trento.

Gentilini, G.; Zamboni, I. (2014). "Considerazioni preliminari per lo studio delle apparecchiature lapidee in contesti castellani trentini in epoca romanica", Archeologia dell'Architettura, XVII, 2012, Firenze, All'Insegna del Giglio.

Marzatico, F. (1988). "L’Alto Garda nella preistoria", in Archeologia dell'Alto Garda: preistoria, età romana, iscrizioni romane, Riva del Garda (TN).

Miani, G. (2011). Castello Penede a Nago. Studio mineralogico-petrografico e fisico-strumentale, ProArte s.n.c., (inedito, Archivio Università di Trento, Dipartimento di Lettere e Filosofia), Vicenza.

Pesavento Mattioli, S. (2000). "Il sistema stradale nel quadro della viabilità dell'Italia nord-orientale", in Buchi, E. ed., Storia del Trentino, II, L'età romana, Bologna, pp. 11-46. 